\title{
Wenn Gleichbetroffene ihr Schicksal in die Hand nehmen
}

\author{
Pascal Pfistera, Conrad Frey \\ a Beauftragter Kommunikation der Stiftung Selbsthilfe Schweiz; ${ }^{b}$ Dr. med., Facharzt Psychiatrie und Psychotherapie/Kinder- und Jugendpsychiatrie \\ und Mitglied der Begleitgruppe des Projektes "Selbsthilfefreundliche Gesundheitsinstitutionen» von Selbsthilfe Schweiz
}

Heute gibt es in der Schweiz doppelt so viele Selbsthilfegruppen (SGH) wie noch vor 15 Jahren. Entgegen dem Klischee der geselligen Plauderrunde spielen diese eine wichtige Rolle im Gesundheits- und Sozialwesen. Eine neue Studie zeichnet ein umfassendes Bild der schweizerischen Selbsthilfelandschaft und formuliert Handlungsempfehlungen an die Akteurinnen und Akteure. Eine Empfehlung sind «selbsthilfefreundliche Gesundheitsinstitutionen».

Selbsthilfegruppen sind der zentrale Untersuchungsgegenstand einer neuen Studie der Universität Lausanne und der Hochschule Luzern Soziale Arbeitdurch folgende Merkmale gekennzeichnet: (a) Die Mitglieder sind durch das gemeinsame Problem selber betroffen, (b) Ziel ist die wechselseitige Hilfe in Bezug auf das gemeinsame Problem, (c) es finden regelmässige Treffen statt, (d) die Mitgliedschaft ist freiwillig, (e) die Verantwortung für und die Entscheidung über die Gruppe liegt ausschliesslich bei den Mitgliedern, (f) es wird eine möglichst gleichberechtigte Zusammenarbeit innerhalb der Gruppe angestrebt und (g) es besteht keine Gewinnorientierung. Das Forschungsteam hat über 1000 Kontaktpersonen von Selbsthilfegruppen ein Fragebogen zugestellt. Zusätzlich wurden sechs Fokusgruppen-Interviews mit 46 Teilnehmenden aus den Kantonen Basel-Stadt, Bern, Jura, Schwyz, Tessin und Waadt und zwölf Gespräche mit Fachpersonen aus dem Sozial- und Gesundheitsbereich geführt. Daraus werden Schlussfolgerungen zu Bedeutung, Nutzen und Grenzen der Selbsthilfe gezogen und Handlungsempfehlungen für verschiedene Akteure des Gesundheits- und Sozialwesens gegeben.

\section{Entwicklungstendenzen der Selbsthilfe}

Lanfranconi L. M. Stremlow J., Ben Salah H. Knüsel R. Gemeinschaftliche Selbsthilfe in der Schweiz. Bedeutung Entwicklung und ihr Beitrag zum Gesundheits und Sozialwesen. Bern: Hogrefe, August 2017. $\rightarrow$ Die Studie kann bestellt werden unter www.selbsthilfeschweiz. ch/studie hilfe Schweiz erfasst über 2500 Selbsthilfegruppen mit etwa 43000 Teilnehmenden. Das sind 32 Teilnehmende pro 100000 Einwohner. 2004 waren es noch 24 . Am höchsten ist dieser Wert mit 55 in der Region Stadt Zürich. Das Wachstum hängt auch massgeblich mit der Neugründung von vier neuen Selbsthilfezentren im Tessin, der Waadt, im Jura und in Neuenburg zusammen. Es besteht aber nach wie vor ein grosses Wachstumspotential. In Deutschland gibt es zum Vergleich pro Kopf etwa zwei- bis dreimal so viele Selbsthilfegruppen.

Drei Viertel dieser Gruppen können dem Gesundheitsbereich zugeordnet werden, ein Viertel sozialen Themen. Somatische Krankheiten betreffen $40 \%$ der Gruppen, Suchtprobleme 20\% und psychische Krankheiten 17\%. Im Vergleich zur Erhebung 2004 wurden 69 neue Themen identifiziert. Bei den Gesundheitsthemen führen Demenz (21 neue SHG), rheumatoide Arthritis (15 neue SHG) sowie bipolare Störungen, Borderline und Zwangsstörungen (je 10 neue SHG) die Liste der Neugründungen an. Im Bereich der sozialen Themen ist es die Einsamkeit (28 SHG).

\section{Vertiefte Zusammenarbeit mit Gesund- heitsinstitutionen}

Die Studie befasst sich auch mit der Organisationsform und Ressourcenausstattung von Selbsthilfe Schweiz und den Selbsthilfezentren, mit ihrer Vernetzung mit Kooperationspartnern und Institutionen des Sozial- und Gesundheitswesens. Selbsthilfe Schweiz pflegt als Mitglied der Gesundheitsligen-Konferenz (GELIKO) und über die Netzwerkkooperation «Nationale Partnerschaften Gemeinschaftliche Selbsthilfe» eine institutionalisierte Zusammenarbeit mit anderen 
Fachstellen, Gremien und verschiedenen Bundesämtern. Die Zentren haben als Drehscheibe für Betroffene und Selbsthilfegruppen eine wichtige Funktion bei der Verbreitung der Selbsthilfebewegung in der Schweiz. Die häufigsten Kooperationspartner dieser Zentren sind psychiatrische Kliniken/Dienste, Sozialberatungsstellen, Behinderten- sowie Selbsthilfeorganisationen und Gesundheitsligen. Die vertiefte Zusammenarbeit der Selbsthilfezentren mit Spitälern und psychiatrischen Diensten sollte im Rahmen der psychosozialen Gesundheit eine wichtige Rolle bei der Stärkung von Betroffenen einnehmen - insbesondere bei seltenen oder tabuisierten Themen. Zu diesem Zweck arbeitet Selbsthilfe Schweiz seit diesem Jahr gemeinsam mit drei Selbsthilfezentren am Projekt «selbsthilfefreundliche Gesundheitsinstitutionen». Eine Infobroschüre dazu kann über info[at]selbsthilfeschweiz.ch bestellt werden.

Die Empfehlungen der Studie richten sich auch direkt an Leistungserbringerinnen und Fachpersonen des Gesundheitswesens. Übereinstimmend mit den befragten Fachpersonen und den Akteuren aus der gemeinschaftlichen Selbsthilfe wird es als besonders gewinnbringend erachtet, wenn die Fachpersonen die Möglichkeiten und Leistungen von Selbsthilfegruppen besser kennen würden. Dies könnte durch ein gezieltes Thematisieren der Gruppen in der Aus- und Weiterbildung von Fachpersonen erreicht werden. Zudem wäre es nützlich, wenn die Fachpersonen in ihrem Arbeitsumfeld vermehrt potenziell interessierte Personen über die Angebote und Leistungen der gemeinschaftlichen Selbsthilfe informieren. Die Selbsthilfegruppenteilnehmenden, besonders jene der AngehörigenGruppen, fordern eine direktere Zusammenarbeit. Damit soll eine bessere Ausrichtung der Interventionen erreicht werden. Die Interaktionen werden als positiv betrachtet, wenn gegenseitiges Verständnis und Respekt gegenüber den Anliegen und Zielen beider Seiten herrschen. Gleiches gilt, wenn eine Komplementarität der Beiträge mit einem echten Vorteil für beide Seiten sowie ein wechselseitiger Austausch bestehen. Um negative Interaktionen zu vermeiden, sollten Beurteilungen und Wettbewerb weggelassen und die entsprechenden Rollen zwischen den Fachpersonen und Selbsthilfegruppen definiert werden. Auch sollen die Fachpersonen ihre Interventionen und die herkömmlichen Arbeitsmodalitäten so anpassen, dass die Gruppen die ihnen zukommende Rolle erfüllen und entsprechend handeln können.

\section{Nutzen und Grenzen}

Die Wirkungen, welchen die Mitglieder einer Teilnahme beimessen, entsprechen den von ihnen er- wähnten Bedürfnissen und werden von den Fachpersonen nahezu vollumfänglich bestätigt. Durch die Teilnahme an einer SHG fühlen sich die Mitglieder besser. Sie haben weniger Schuldgefühle und fühlen sich mit einer schwierigen Situation nicht allein gelassen. Praktische Lösungen können gefunden werden. Für einige bedeutet die Teilnahme auch, die Situation selbst in die Hand zu nehmen und sie nicht einfach zu erleiden. Eine Teilnehmerin aus Basel: «Man befindet sich in einer Sackgasse, wenn man nicht mehr weiss, was machen. Fachpersonen können zwar helfen, aber die emotionale Seite ist in den Gruppen viel präsenter.» Wirkung entfaltet die Beteiligung auch auf die Beziehung mit anderen. Die Gruppenteilnehmenden verbessern ihre Beziehungen mit ihren Nahestehenden. Es werden untereinander neue Beziehungen geknüpft. Die Beziehungen zu den Fachleuten verbessern sich durch gezielteres Nachfragen und das vermehrte Treffen von eigenen Entscheidungen. Zu den Grenzen und Schwierigkeiten gehören gruppeninterne Probleme (zu geringes Engagement, ausbleibende Neumitglieder), Probleme mit der Funktionsweise und zunehmende Individualität (Nichteinhalten der Regeln, schwierige Charaktere) sowie logistische und technische Grenzen (Schwierigkeit, geeignete Räumlichkeiten $\mathrm{zu}$ finden und entfernt wohnende Personen $\mathrm{zu}$ Treffen zusammenzuführen, knappe Finanzierung). Aus Sicht der Fachpersonen kommen auch Wirkungen auf die Gesellschaft als Ganzes hinzu. Die Unterstützung von Selbsthilfegruppen sollte als gesundheitlich relevante Präventionsmassnahme und auch im Sinne der Nachsorge gefördert werden. Die befragten Fachpersonen vertreten die Ansicht, dass die Selbsthilfe einen ergänzenden Ansatz zu den professionellen Interventionen darstellt, aber gegenwärtig nicht ausreichend von den medizinischen und sozialen Bereichen gefördert wird, obwohl auch von einem finanziellen Nutzen in Bezug auf die Gesundheitskosten auszugehen ist. Die Tatsache, dass die meisten Selbsthilfegruppen wenig institutionalisiert sind, bedeutet nicht, dass sie bezüglich ihrer behandelten Problematiken losgelöst von den Fachbereichen Medizin, Psychiatrie oder Sozialwesen funktionieren. Die nahezu gleiche Einschätzung der Wirkungen zeigt, dass ein gewinnbringender Austausch zwischen Selbsthilfegruppen und den fachlichen Bereichen herrscht. Die Zusammenarbeit mit den Fachleuten ist aber auch nicht ganz frei von Spannungen. Die Ermächtigung (Empowerment) der Gruppenmitglieder in Gestalt der Anerkennung und der Positionierungsmöglichkeit ermöglicht es ihnen, Gelegenheiten zu schaffen, ihr Erfahrungswissen gegenüber der professionellen Vertreterinnen und Vertretern des Gesundheits- und Sozialwesens zu behaupten. 